Result We analysed a total of 21,335 tests in intervention and 13,992 in control clinics. In clinics with 12-15 months intervention period (11 clinics), annual testing has increased by $47 \%$ (from $15.1 \%$ to $22.3 \%$ ) in females, $88 \%$ (from $6.3 \%$ to $11.9 \%$ ) in males and $61 \%$ overall (from $11.6 \%$ to $18.7 \%$ ). After increasing from $7.9 \%$ to $11.8 \%$ in the first 12 months, control clinic testing dropped to $9.7 \%$ in 2012. The relative risk of testing in intervention compared with control clinics is 1.9 (95\% CI: 1.8, 2.0). Of 2876 testing positive, $38 \%$ retest within 3 months and of those testing negative, 22\% retest within 12 months in both trial arms.

Conclusions Nearly twice as many are being tested in the intervention and testing rates are increasing. The high participation rate of clinics in all towns puts the study in a strong position to determine if a pragmatic intervention can reduce chlamydia prevalence.

\section{P5.018 IMPROVED DIAGNOSIS OF NON-NEOPLASTIC CONDITIONS OF CERVIX BY LIQUID BASED CYTOLOGY, CELLBOCK IN COMPARISON WITH CONVENTIONAL PAP SMEAR METHOD}

doi:10.1136/sextrans-2013-051184.1063

N N Manoli, N Manoli, S Kulkarni, A P Chandrashekar. JSS Medical College, Mysore, India

Background The Papanicolaou (Pap) smear is the most successful screening test for carcinoma of cervix, mainly as a result of its simplicity, low cost, low false-negative rate and its early detection of preneoplastic lesions. It is also used to diagnose several infectious agents that manifest with specific cytologic features.

The diagnoses of candida, trichomonas, herpes simplex virus, HPV and bacterial vaginosis can be reliably rendered on Pap smears.

Conventional pap smear (CPS) has its limitations. False negatives in CPS may be related to inadequate sampling, inadequate transfer of the sample onto the glass slide or deficiencies in the microscopic assessment of the slide. To overcome these problems, a new slide preparation method namely the Manual Liquid Based Cytology (MLBC) was introduced, where cells are uniformly dispersed by a membrane, from a suspension of cells in a polymer solution.

MLBC helps in detecting number of infestations like Candida, Leptothrix, HPV with koilocytic atypia and bacterial vaginosis, as observed by many authors and us also.

MLBC, which we are following is inexpensive, cost effective method which we have adapted and are comparing it with CPS for its adequacy and utility. The other advantages, is that the residual specimens can be used for ancillary testing like immunocytochemistry by cell block preparation.

Results Number of cases of bacterial vaginosis diagnosed by CPS were 8, LBC 9 and cell block were zero. trichomonas were 4, by CPS andLBC,2bycellblock., herpeswas 1 byall threemethods, inflammatory (NILM) cases were 22 by CPS, 26 by LBC, 12 by cell block. Inadequate smears were 5 by CPS, NIL by LBC, 25 by cell block

Conclusion CPS and LBC with cell block are useful methods in detection of non neoplastic conditions of cervix.

LBC has increased the diagnostic accuracy by $13 \%$ in our study.

\section{P5.019 STRATEGIES INFLUENCING DID-NOT-ATTEND (DNA) RATES IN SEXUALLY TRANSMITTED INFECTION (STI) CLINICS: A RANDOMISED CONTROLLED TRIAL (RCT) ASSESSING THE EFFECTIVENESS OF A VERBAL WARNING MESSAGE GIVEN AT APPOINTMENT BOOKING}

doi:10.1136/sextrans-2013-051184.1064

D Vijeratnam, P Satchithananthan, J Davies, R Patel, D Rowen, E Foley. Sexual Health Department (GUM), Royal South Hants Hospital, Southampton, UK

Background Missed clinic appointments cause significant wastage of health resources worldwide. The majority of cases who
DNA appointments are young and of lower socio-economic status and, conversely, are most likely to carry STIs. Several interventions aimed at reducing DNA appointments such as reminder SMS texts and letters have been studies internationally. Our UK STI department uses interventions such as monthly displays of DNA appointments, SMS reminders and removal of booking privileges to reduce DNA rates. The objective of this study was to assess whether introducing a verbal missed appointment warning at the time of booking has an effect on subsequent DNA and cancellation rates.

Method 108 patients over 16 years old were recruited in this RCT to show a $25 \%$ difference between the groups. Within the first arm the patients were advised that if they did not cancel their appointments in advance and subsequently DNA'ed then they would be obliged to attend a walk-in service in the future. In the second arm the warning was removed. The DNA rates from these two arms were compared initially to each other and then to other previously studied interventions.

Results Interim analysis of results shows a fall in the DNA rates with those who received a verbal warning message at the time of booking matching SMS text and letter reminders rates seen in published data.

Conclusion With financial constraints affecting STI clinics worldwide, it is essential to consider low-cost methods aimed at reducing DNA rates. Whilst we have shown a warning message to be effective, it is also inexpensive and introduction of such a warning system would be beneficial in resource-limited settings.

\section{P5.020 DRIED BLO0D SAMPLES ON PHILTRE PAPER COULD BE A SOLUTION FOR SCREENING OF HIV AND SYPHILIS IN HARD TO REACH POPULATIONS}

doi:10.1136/sextrans-2013-051184.1065

1J F Bencomo-Gomez, 'G Falero-Diaz, ${ }^{1}$ M A 0 Appolinario, ${ }^{2} \mathrm{~A}$ G P Ferreira, ${ }^{2} \mathrm{E} D$ Silva, ${ }^{1}$ A J Werneck de Castro. IInstituto Vital Brazil - Biomarc, Niterói, Brazil; ${ }^{2} I n s t i t u t o$ Oswaldo Cruz - Bio-Manguinhos, Rio de Janeiro, Brazil

Background The high incidence of HIV and syphilis is a health problem in low-income, isolated and large populations, and one of the most affected sectors is the one of pregnant women. Therefore it is necessary to develop and implement methods and procedures to detect and follow new positive cases for those diseases. The aim of this study was to demonstrate the usefulness of the collection of dried blood on philtre paper for the diagnosis of HIV and syphilis in pre-natal screening programmes in areas of difficult access

Methods Dried blood samples on philtre paper were collected and sent to them by mail without any special condition to the laboratory to be processed. They were studied for the presence of antibodies against HIV or syphilis antigens by ELISA. Reactive samples were confirmed by employing a rapid immunochromatography test to both diseases. Finally, classification between HIV type 1 or 2 was performed by rapid immunoblot test.

Results Of the samples tested for HIV by ELISA, the $0.37 \%$ was positive, so 44 samples showed specific antibodies. They all confirmed when rapid immunochromatography test was used. In addition, $100 \%$ of the samples classified as HIV type 1 by using the rapid immunoblot test. In the case of the samples studied for syphilis by ELISA, the positive ones were the $3.12 \%$, being confirmed by the rapid test all 41 positive samples.

Conclusion It was shown that the use of dried blood collected on philtre paper proved to be a cheap, safe and quick way for the screening of infectious diseases, such as HIV and syphilis, in populations as sensitive as pregnant women. It was also found that, although the test of choice for this type of work is the ELISA, other tests such as rapid tests can be used as a confirmatory test 